\title{
Micro-Mechanism Research into Molecular Chains Orientation Synergistically Induced by Carbon Nanotube and Shear Flow in Injection Molding
}

\author{
Meili Zhang ${ }^{1,2}$ and Yong Xin ${ }^{1, *}$ \\ 1 College of Mechanical and Electrical Engineering, Nanchang University, Nanchang 330031, China; \\ zhangmeili@jju.edu.cn \\ 2 School of Mechanical \& Materials Engineering, Jiujiang University, Jiujiang 332005, China \\ * Correspondence: xinyong_sh@sina.com
}

Received: 23 December 2019; Accepted: 18 January 2020; Published: 20 January 2020

\begin{abstract}
For the degree of orderly arrangement of the molecular chains at the interface of nanocomposites, the static and sheared polyethylene (PE)/carbon nanotube (CNT) models and the sheared pure PE model were constructed, and molecular simulation experiments were carried out in comparison. The micro-mechanism of molecular chains orientation, synergistically induced by the carbon nanotube and shear flow in injection molding, was discussed by analyzing the radius of gyration, molecular chain motion, conformation evolution of molecular chains, bond orientation parameter, interface binding energy and atom distribution. The results show that, for the static composite system, the conformation adjustment of PE molecular chains induced by CNT is limited due to the hindrance from the surrounding chains. Thus, the orientation and radius of gyration of molecular chains increase slightly. For the sheared pure PE system, the orientation induced by shear flow is unstable. After the cessation of shear, the molecular chains undergo intense thermal movement and relax quickly. The disorientation is obvious, and the radius of gyration decreases considerably. It is worth noting that for the sheared composite system, shear flow and the CNT have a synergistic effect on the orientation of the molecular chains, which is due to the adsorption effect of the CNT on shear-induced oriented chains and the inhibition effect of CNT on the relaxation of shear-induced oriented chains. Thus, the orientation stability of PE chains is greatly improved, and interface crystallization is promoted. Moreover, because of the more regular arrangement of molecular chains in the sheared composite system, more $\mathrm{H}$ atoms and $\mathrm{C}$ atoms are close to the surface of the CNT, which increases the van der Waals force, and correspondingly increases the interface binding energy.
\end{abstract}

Keywords: molecular chains orientation; synergistic effect; micro-mechanism; injection molding

\section{Introduction}

Since the discovery of carbon nanotubes (CNTs) by lijima in 1991 [1], their excellent mechanical [2,3], thermal $[4,5]$, and electrical properties [6,7] have promoted their use as fillers in composites. Especially after the first preparation of epoxy resin/CNT by Ajayan et al. [8], nanocomposites have been extensively studied. Polyethylene (PE) is an important plastic material, which has the advantages of a wide range of raw materials, low price and stable chemical properties, but its service life is reduced due to low strength, easy deformation, and heat resistance [9]. Thus, it is necessary to study PE nanocomposites. In nanocomposites, the interface plays the role of transmitting the load from polymer to CNTs under the action of external force; therefore, the interface interaction is crucial. The orderly arrangement degree of the polymer molecular chains on the surface of the CNT directly affects the interface crystallization 
behavior and interface interaction [10,11]. Therefore, the orientation and conformation of molecular chains on the surface of the CNT can be studied to regulate and control the interface structure and improve interface interaction.

In recent years, the molecular simulation method has been applied to the study of nanocomposites [12-16], and many molecular simulation studies have been conducted on the molecular chain orientation and conformation evolution induced by CNT. Yang et al. analyzed the orientation process of multiple short PE chains induced by the CNT [17]. Yang et al. studied the orientation process of a single PE chain induced by the CNT, and found that the ordered structures formed by PE chains with different lengths were all almost parallel to the axis of the CNT, with a shorter chain forming an ordered single-layer structure, and a longer chain forming an ordered multi-layer structure [18]. Liu et al. studied the adsorption and diffusion behavior of molecular chains on the surface of semi-metallic and semiconductor CNTs. The results showed that the adsorption position and conformation of molecular chains are mainly related to the temperature and the radius of the CNT, not the type of CNT [19]. Karatrantos et al. found that only the structure of local polymer molecular chains close to the CNT was more orderly, while the overall chain conformation did not change significantly [20]. Wei et al. found that the molecular chains in the adsorption layer tended to be parallel to the axis of the CNT, and improvement of the structure order degree strengthened the nanocomposites [11]. These studies mainly focused on the individual effect of the CNT inducing the orientation of molecular chains under static conditions. In addition, Yu et al. compared and discussed the degree of orientation and the interface crystallization behavior when the specific initial orientation angles of molecular chains and the CNT were $0^{\circ}, 45^{\circ}$, or $90^{\circ}$ [10].

However, shear flow inevitably occurs in the melt during the injection molding of nanocomposites [21,22]. For the thermoplastics with a linear flexible molecular chain structure, under different process conditions of injection molding, the molecular chains will have different degrees of orientation under the induction of shear flow, thus affecting the degree and morphology of crystallization at the interface. The molecular mechanism of the orientation of multiple random molecular chains in melt under the combined effect of shear flow and the CNT has not been reported. In this study, the dynamic evolution process of the conformation of molecular chains at the interface of nanocomposites was more intuitively observed using molecular simulation technology. The micro-mechanism of molecular chains orientation, synergistically induced by carbon nanotube and shear flow, is discussed. Meanwhile, the finite behavior of the CNT inducing the conformation adjustment of multiple random PE molecular chains under static conditions, and the rapid relaxation phenomenon of a sheared pure PE system after the cessation of shear, were analyzed. Additionally, the principle whereby the interface binding energy of a sheared composite system is higher than the static composite system was also studied. The findings provide theoretical guidance for regulating and controlling the interface structure and improving the interface interaction of nanocomposites by controlling the processing condition.

\section{Model Construction and Simulation Experiment}

Since the influence of the length of PE chain, the radius and chirality of the CNT on molecular chains orientation, and the aspect ratio of the $\mathrm{CNT}$ on the reinforcement effect of composite materials, had been investigated $[18,19,23]$, in the constructed PE/CNT model, each PE chain contained 120 repeating units, and the single-walled carbon nanotube was $(10,10)$ armchair type terminated by hydrogen atoms at both ends, with a length of $241.03 \AA$. In the real system of PE/CNT nanocomposites, the content of the CNT was low and most of them were PE molecular chains, so twenty amorphous PE chains were randomly distributed on the surface of the CNT, as shown in Figure 1a, which depicts the initial model of the composite system. After structural optimization, this initial model ran for $100 \mathrm{ps}$ at $550 \mathrm{~K}$ to simulate the melting process. Figure $1 \mathrm{~b}$ shows the initial conformation after melting under static conditions. Then, the initial conformation after melting was shear loaded along the $z$-axis and ran for $700 \mathrm{ps}$ at a shear rate of $0.01 \mathrm{ps}^{-1}$ to simulate the shear flow of melt during injection molding. The conformation after shear is shown in Figure 1c. Both PE molecular chains and the CNT tended to 
be parallel to the shear flow direction, which is consistent with the simulation results reported by Wang and Zhou et al. [24,25]. To compare the change in the morphology of the molecular chains between the sheared pure PE system and sheared composite system after the cessation of shear, the oriented PE chains induced by shear flow in the composite system were selected as the initial conformation of the pure PE system after shear, as shown in Figure 1 d.

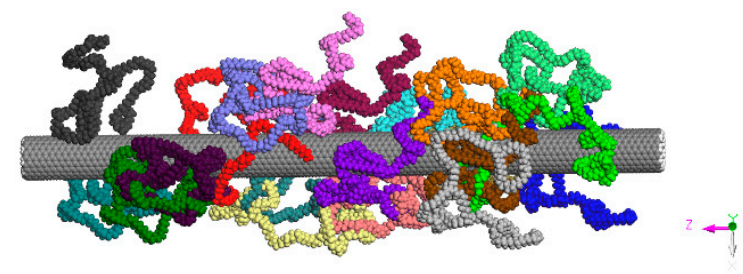

(a)

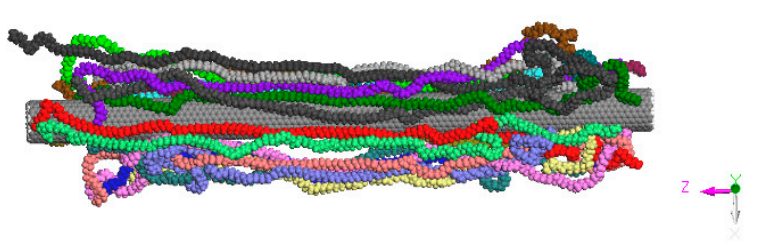

(c)

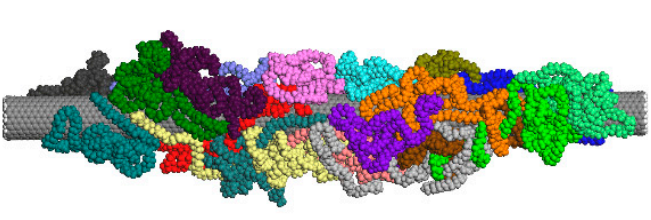

(b)

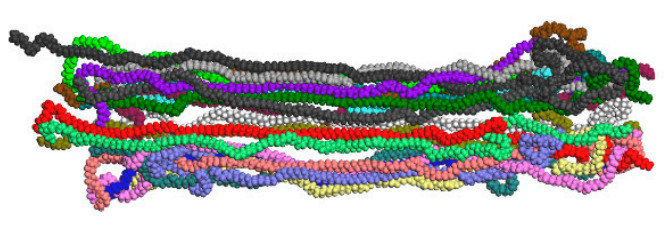

(d)

Figure 1. Simulation models. (a) Initial model of composite system; (b) Initial conformation of composite system under static conditions; (c) Initial conformation of the composite system after shear; (d) Initial conformation of pure polyethylene (PE) system after shear.

Next, the static and sheared composite systems and the sheared pure PE system were cooled in stages, from 500 to $380 \mathrm{~K}$, respectively, and cooling at each stage was $30 \mathrm{~K}$. Yang et al. showed that whether the CNT was fixed had little effect on the simulation results [26], so in order to save simulation time, the position of carbon atoms of the CNT in the system was fixed. The simulated ensemble was an NVT ensemble (constant number, constant volume, and constant temperature) with a total simulation time of 500 ps. The final conformation of each stage served as the initial conformation of the next stage. Material Studio software was used for calculation. The Nose-Hoover temperature control method was adopted to keep the system temperature constant. The COMPASS force field [27], suitable for the calculation of organic and inorganic materials, and the Velocity Verlet integral algorithm, with excellent performance and wide application, were adopted, and the calculation time step was $1 \mathrm{fs}$. The cutoff distance was $12.5 \AA$.

\section{Results and Discussion}

\subsection{Radius of Gyration}

Polymer molecular chains have multiple conformations due to the internal rotation of single bonds. Under the action of thermal motion, the molecular conformation changes constantly, and the molecular size and morphology change accordingly. The mean square radius of gyration can be used to characterize the size of the polymer molecular and the morphological distribution characteristics of molecular chains. It is defined as the mass average of the square of the distance from all atoms to their center of mass, which can be expressed as

$$
R(g)^{2}=\sum_{i=1}^{n} m_{i} r_{i}^{2} / \sum_{i=1}^{n} m_{i}
$$


where $R(g)$ is the radius of gyration, $m_{i}$ is the mass of the $i$ th atom, and $r_{i}$ is the distance from the $i$ th atom to the center of mass of the molecular chain.

According to the distribution curve of the radius of gyration in Figure 2 and the evolution curve of the mean radius of gyration in Figure 3, the distribution interval (10.8, $27.46 \AA$ ) of the radius of gyration of PE molecular chains in the static composite system is small, and the mean radius of gyration increases gently. This also shows that PE molecular morphology changes little and the adjustment induced by the CNT is limited in the static composite system, while the distribution range (53.84-74.86 $\AA$ ) of the radius of gyration of PE molecular chains in the sheared composite system is markedly increased. The mean radius of gyration decreases a little initially, and then increases gradually. This also indicates that elastic shrinkage occurs to some extent, due to the viscoelastic properties of the molecular chains at a higher melt temperature after the cessation of shear. Subsequently, the molecular chains further extend under the action of the CNT. In particular, the distribution curve of the radius of gyration of the sheared pure PE system moves to the left, relative to the sheared composite system, and the distribution range decreases significantly. Moreover, the mean radius of gyration decreases sharply within 100 ps. This shows that the atoms migrate closer to the center of mass of the molecular chain at a higher temperature after the cessation of shear, the size of molecular chains decreases considerably, and the degree of bending and winding of the molecular chains rises quickly. Then, the radius of gyration gradually stabilizes with the decreasing temperature.



Figure 2. The distribution curve of the radius of gyration of PE molecular chains after running for 500 ps.

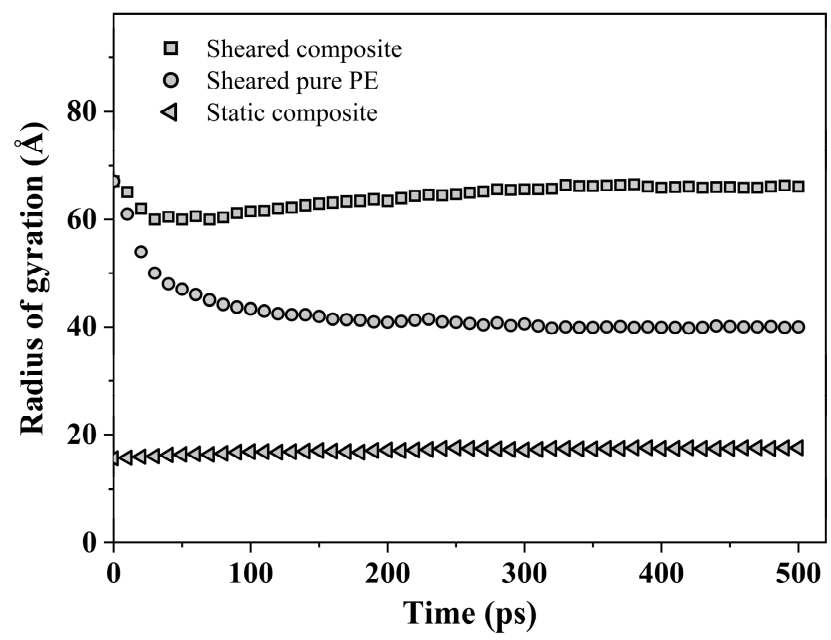

Figure 3. The evolution curve of the mean radius of gyration of PE molecular chains. 
It can also be seen from Figure 3 that the mean radius of gyration (66.2 $\AA$ ) of the PE molecular chains in the sheared composite system is not only higher than the sheared pure PE system and the static composite system, but also higher than the sum of the static composite system (17.5 $\AA$ ) and the sheared pure PE system ( $39.9 \AA$ ). Therefore, compared with the single effect of shear flow and the CNT, the combination of the two has a more obvious effect on the size of the molecular chains.

\subsection{Conformation Evolution of Molecular Chains}

Figure 4 shows the conformation evolution process of PE molecular chains. As can be seen from Figure $4 \mathrm{~b}$, a certain degree of relaxation occurs in the PE molecular chains of the sheared composite system during the initial period after the cessation of shear. However, due to the adsorption effect of CNTs [28], relaxation of the molecular chains is inhibited, which stabilizes the conformation of the molecular chains. The principal axis of the molecular chains is still basically parallel to the Z-axis. Over time, and with decreasing temperature, the CNT induces PE molecular chains to adjust conformation along its axial direction, to increase the orderliness of their arrangement. As shown in Figure 4c, the sheared pure PE system, with the same extension degree as the PE chains in the sheared composite system, undergoes a significant conformation change after the cessation of shear. Without the restriction of the CNT, PE molecular chains relax considerably at a high temperature and rapidly evolve from orientation state to winding state. For the static composite system, as shown in Figure 4a, PE molecular chains are mostly distributed around the CNT in a disordered state at the beginning, and the number of molecular chains is large. Hence, the molecular chain segments are hindered by the surrounding molecular chain segments in the process of the CNT-inducing conformation ordering. Furthermore, for locations where the arrangement of molecular chains around the CNT is slightly sparse, the space for conformation adjustment is a little larger, and the molecular chain has different degrees of folding arrangement. However, for locations where the molecular chains are more densely arranged, the space for conformation adjustment is smaller, and the regularity is even worse.

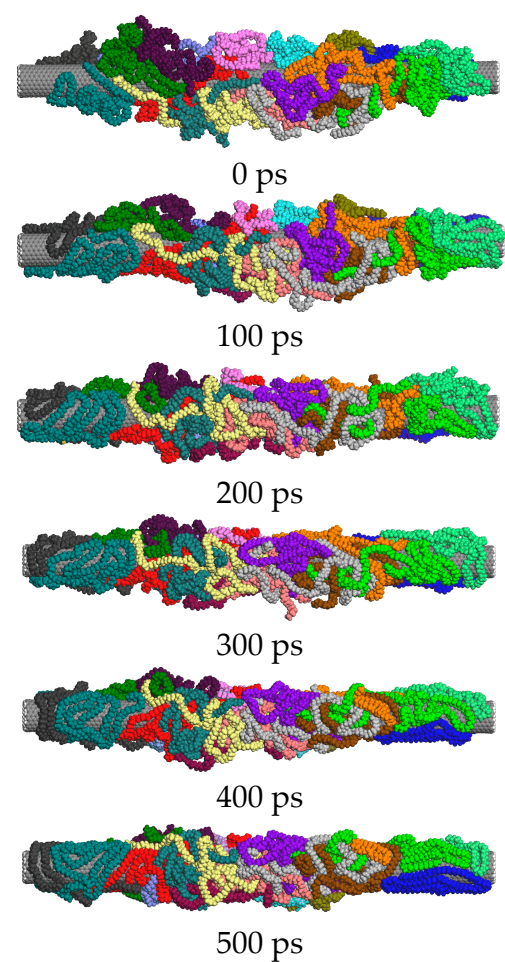

(a)

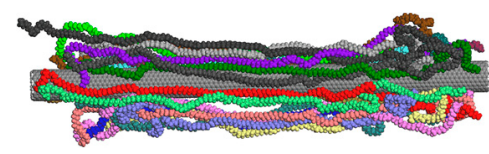

0 ps

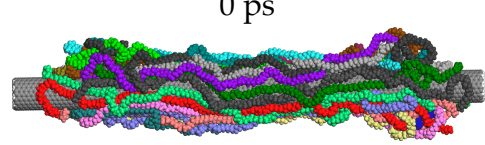

$100 \mathrm{ps}$

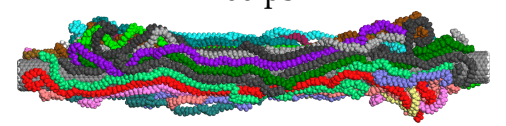

200 ps

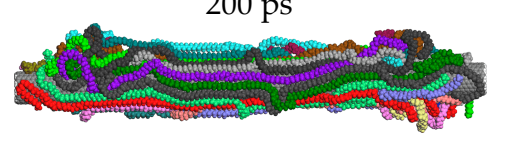

300 ps



400 ps

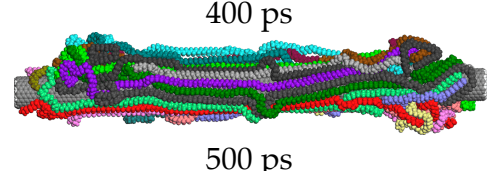

(b)



100 ps

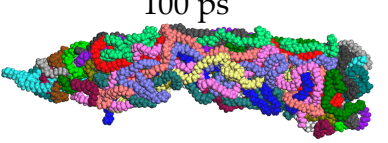

200 ps



300 ps

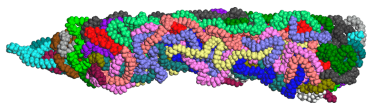

400 ps

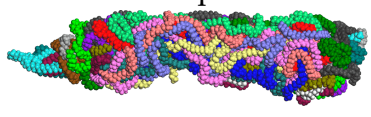

500 ps

(c)

Figure 4. The conformation evolution process of polyethylene (PE) molecular chains. (a) The static composite system; (b) The sheared composite system; (c) The sheared pure PE system. 


\subsection{Molecular Chain Motion}

The motion of the molecular chains is closely related to the conformation evolution of the molecular chains. It is usually characterized by mean square displacement (MSD), which is the mean square value of the increment of the atomic position vector at different times. It can be used to characterize the migration behavior of PE chains, which can be expressed by

$$
M S D=\left\langle\left|r_{i}(t)-r_{i}(0)\right|^{2}\right\rangle
$$

where $r_{i}(0)$ and $r_{i}(t)$ are the positions of the $i$ th atom at the initial time and time $t$, respectively.

According to the change in the trend of $M S D$ with time, as shown in Figure 5, the MSD values of the three curves increase most in their respective $100 \mathrm{ps}$, and the slopes of the curves are highest. According to the Einstein equation, the atomic diffusion coefficient $D$ is one-sixth of the slope of the MSD curve, as shown in Equation (3) [29]. When the temperature is high, the energy of the system is correspondingly high, and the molecular chains easily overcome the migration barrier and cross the region of high potential energy. Thus, the diffusion of molecular chains is promoted, with a high diffusion coefficient and MSD value. Especially for the sheared pure PE system after the cessation of shear, the diffusion coefficient is much larger than those of the composite systems. For a linear chain polymer with good flexibility, the viscous flow activation energy is low. The viscous flow activation energy is the minimum energy required for the molecular chain segments to overcome the barrier and jump from the original position to the adjacent "hole". Therefore, PE molecular chains can easily overcome the viscous flow activation energy, undergo a violent thermal movement, and shorten the relaxation time, which is the time required for the molecular chains to recover from the unstable orientation state to the low-energy winding state through molecular motion. Subsequently, the entanglement barrier is difficult to overcome with decreasing temperature, the relaxation speed decreases significantly, and the slope of the MSD curve decreases rapidly. Thus, the molecular chains migration weakens. However, the MSD of PE chains in the sheared composite system is markedly reduced. This occurs because the molecular chains movement slows down under the action of CNT adsorption, and the stretched state of PE chains is basically maintained. Thus, the molecular chain segments only need minor adjustments, and only migrate and diffuse within a small range. The slope of the MSD curve is very gradual. Compared with the sheared composite system, the molecular chain segments need to be adjusted slightly more under the induction of the CNT, due to the initial molecular chains of the static composite system, which are mainly in a random state. The migration and diffusion of the molecular chains are slightly stronger, and the MSD is slightly larger.

$$
D=\frac{1}{6} \lim _{t \rightarrow \infty} \frac{d}{d t}\left\langle r_{i}(t)-\left.r_{i}(0)\right|^{2}\right\rangle
$$

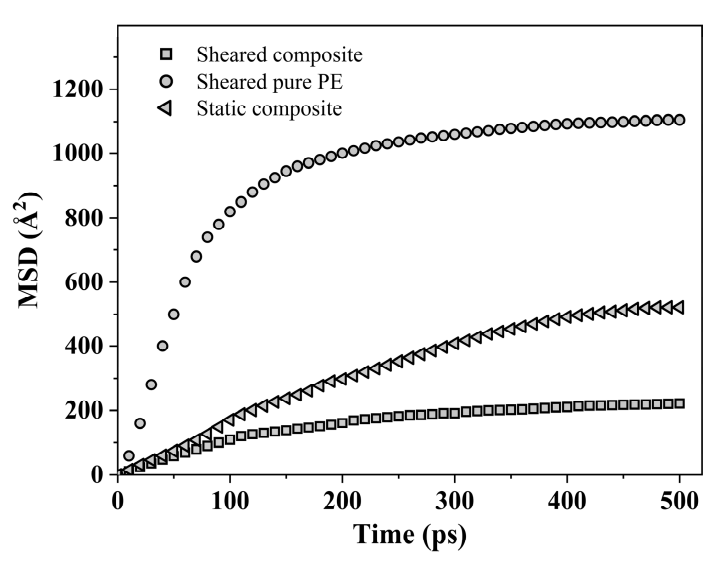

Figure 5. The change in the mean square displacement $(M S D)$ of PE molecular chains over time. 


\subsection{Molecular Chain Orientation}

It can be seen in Figure 4 above that the conformation changes in the molecular chains are accompanied by corresponding changes in the orientation of the molecular chains. In order to quantitatively study the orientation of polymer molecular chains, the bond orientation parameter $S[30]$ is introduced, and the calculation formula is as follows,

$$
S=\frac{1}{N(n-2)} \sum_{m=1}^{N} \sum_{i=3}^{n} \frac{3 \cos ^{2} \psi_{i}^{m}-1}{2}
$$

where $N$ is the number of PE molecular chains, $n$ is the number of $C$ atoms in each PE molecular chain, and $\psi$ is the angle between two adjacent bond segment vectors and the CNT axis.

As shown in Figure 6, the bond orientation parameter of PE molecular chains in the static composite system slowly increases. The slight change further indicates that when the CNT is surrounded by multiple random polymer chains, the orientation of molecular chains induced by the CNT is finite under the restriction of the surrounding chain segments. The conformation evolution diagram also shows that the orientation of these chain segments is inconsistent. For a sheared pure PE system, the orientation induced by shear flow is unstable. The molecular chains are clearly disoriented and the bond orientation parameter is greatly reduced after the cessation of shear. For the sheared composite system after the cessation of shear, the bond orientation parameter decreases a little in a short time and then increases slowly. The degree of disorientation is much lower than that of the sheared pure PE system, which further indicates that the orientation stability of PE chains markedly improves under the adsorption effect of the CNT on shear-induced oriented chains and the inhibition effect of the CNT on the relaxation of shear-induced oriented chains. Moreover, the degree of orientation is further improved under the induction of the CNT.

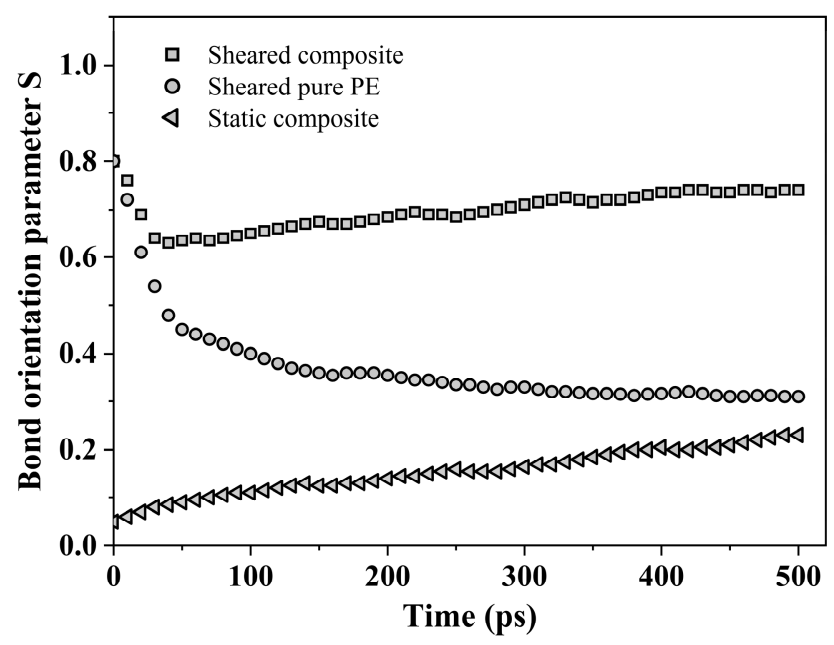

Figure 6. The change curve of the bond orientation parameters of PE molecular chains over time.

Figure 6 also shows that, under the combined action of the CNT and shear flow, the $S$ value (0.74) in the sheared composite system is higher than the linear addition of $S$ values $(0.31)$ in the sheared pure PE and $S$ values (0.23) in the static composite system. This indicates that shear flow and the CNT have a synergistic effect on the induction of orientation of the molecular chains. This is consistent with the analysis results of the radius of gyration. During the cooling process of injection molding, the polymer melt will undergo a transition from the liquid phase to the solid phase, that is, the crystallization process including the formation of a crystal nucleus and the growth of grain. At a higher temperature, the crystal nucleus is easily destroyed by molecular thermal motion, such as in the sheared pure PE system mentioned above. For the sheared composite system, the molecular chains maintain a stable 
orientation under the action of the CNT. Since the molecular chains with a high orientation degree induce not only the orientation of the molecule chains around them, but also are closer to a lattice structure, the molecular chains have less kinetic barriers to cross, which is conducive to the formation of the crystal nucleus. They have a better crystallization ability than the chains with low orientation, thus promoting the interface crystallization of the composite system.

\subsection{Interface Binding Energy and Atom Distribution}

The interface binding energy in this composite system is a measure of the interaction energy between PE molecular chains and CNT, which can be expressed as

$$
E_{\text {bind }}=-E_{\text {inter }}=-\left[E_{P E / C N T}-\left(E_{C N T}+E_{P E}\right)\right]
$$

where $E_{\text {bind }}, E_{\text {inter }}, E_{P E / C N T}, E_{C N T}$, and $E_{P E}$ are the interface binding energy, the interface interaction energy, the total energy of the composite system, the energy of CNT, and the energy of PE, respectively.

As shown in Table 1, the interaction energy between PE molecular chains and the CNT is equal to the non-bond interaction energy under both static and sheared conditions, which indicates that PE molecular chains and the CNT interact through non-bonds. Since the electrostatic interaction is small, the strong and weak interface binding energy is mostly determined by van der Waals interaction. Moreover, the interface binding energy of the sheared composite system is higher than in the static composite system.

Table 1. Energy of the composite system after running for $500 \mathrm{ps}\left(\mathrm{kJ} \cdot \mathrm{mol}^{-1}\right)$.

\begin{tabular}{ccccccccc}
\hline \multirow{2}{*}{ Energy } & \multicolumn{9}{c}{ Static } & \multicolumn{4}{c}{ Shear } \\
\cline { 2 - 9 } & $\mathbf{P E} / \mathrm{CNT}$ & $\boldsymbol{P E}$ & $\boldsymbol{C N T}$ & $\boldsymbol{E}_{\text {inter }}$ & $\boldsymbol{P E} / \boldsymbol{C N T}$ & $\boldsymbol{P E}$ & $\boldsymbol{C N T}$ & $\boldsymbol{E}_{\text {inter }}$ \\
\hline$E_{\text {total }}$ & 817,566 & -4205 & 833,057 & $-11,286$ & 746,293 & $-73,773$ & 832,769 & $-12,703$ \\
$E_{\text {non-bond }}$ & -4427 & $-10,839$ & 17,698 & $-11,286$ & $-14,211$ & $-19,322$ & 17,814 & $-12,703$ \\
$E_{\text {vdW }}$ & 1643 & -4552 & 17,443 & $-11,248$ & -5037 & -9936 & 17,556 & $-12,657$ \\
$E_{\text {electrostatic }}$ & -6065 & -6286 & 259 & -38 & -9175 & -9388 & 259 & -46 \\
\hline
\end{tabular}

To further understand the effect of the orderly arrangement degree of molecular chains on interface binding energy, Figures 7 and 8 respectively show the radial distribution function (RDF) of the hydrogen $(\mathrm{H})$ atoms and carbon $(\mathrm{C})$ atoms of PE molecular chains in the composite system under static and sheared conditions. It can be seen that the peaks of the curves become increasingly obvious over time and with decreasing temperature. At 500 ps, the position of the peak of $C$ atoms is $4.5 \AA$ from the surface of the CNT, and the position of the first peak of $\mathrm{H}$ atoms is $3.2 \AA$ from the surface of the CNT, both of which are within the range of van der Waals forces $(r=3.1-5.0 \AA)$. When the distance exceeds $5.0 \AA$, the van der Waals interaction is weak. Furthermore, the peak value of the sheared composite system is higher than that of the static composite system, which further indicates that the orderly arrangement degree of molecular chains under shear condition is higher than under static condition. The molecular chains in the sheared composite system are arranged more closely, so more $\mathrm{H}$ and $\mathrm{C}$ atoms are close to the surface of the CNT. Therefore, the van der Waals force increases, and the interface binding energy increases correspondingly. This also indicates that the interface interaction between PE molecular chains and CNTs can be enhanced to some extent through interface crystallization. 


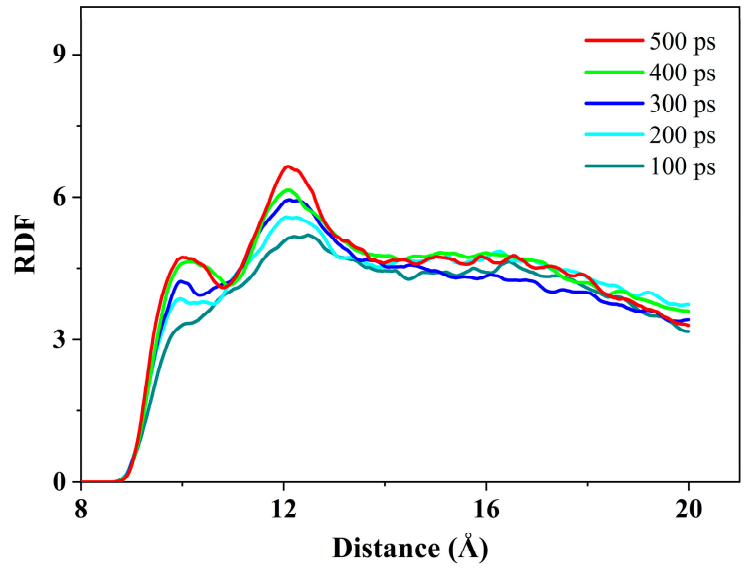

(a) The static composite system

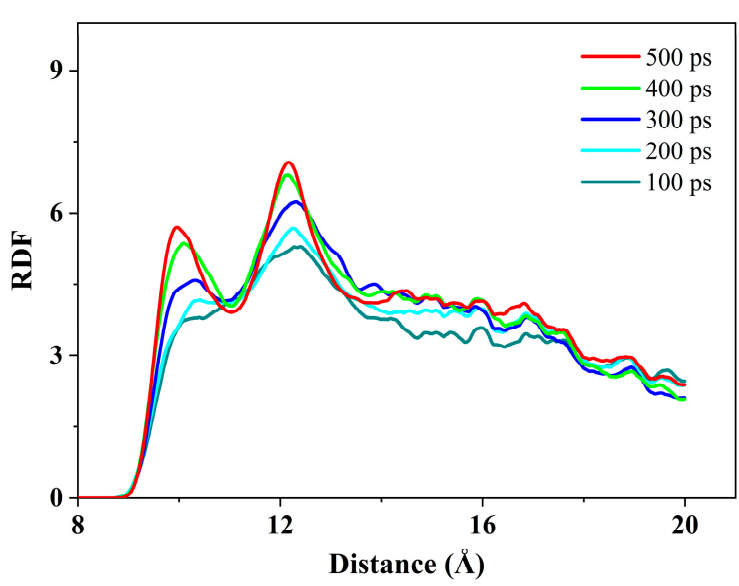

(b) The sheared composite system

Figure 7. The radial distribution function (RDF) of hydrogen atoms in PE chains along the carbon nanotube (CNT) radial direction.

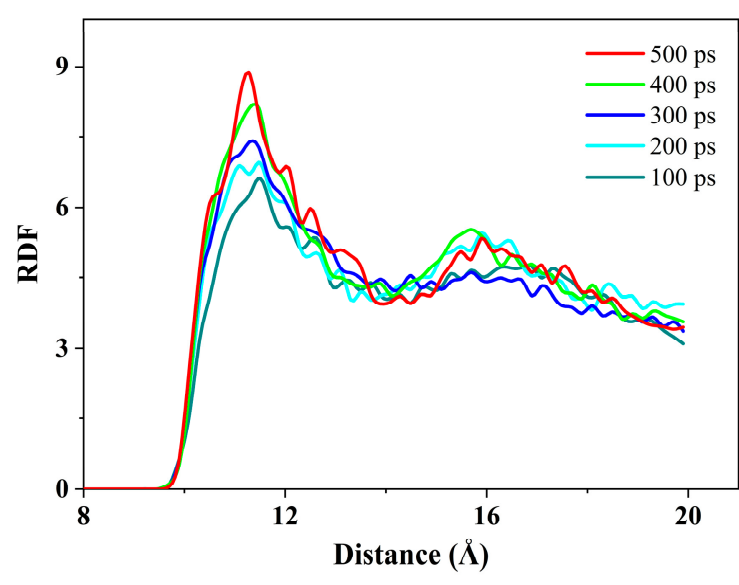

(a) The static composite system

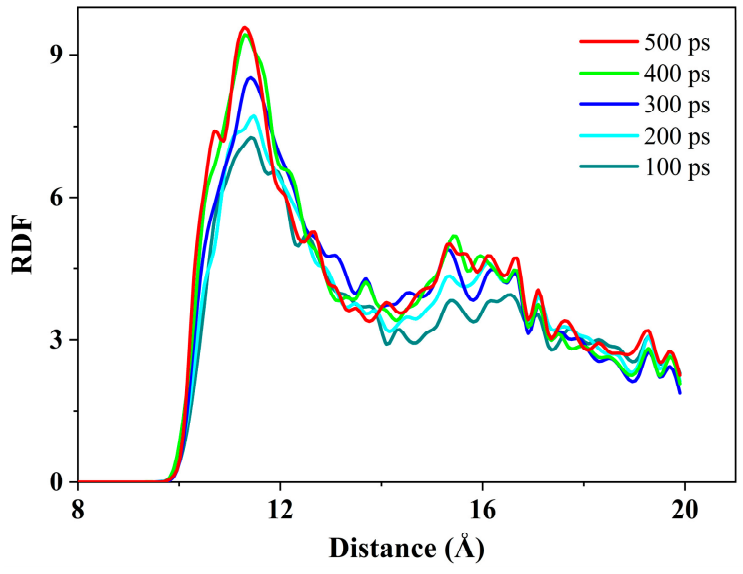

(b) The sheared composite system

Figure 8. RDF of carbon atoms in PE chains along the CNT radial direction.

\section{Conclusions}

(1) In the static composite system, when the CNT is surrounded by multiple random polymer chains, the CNT has a tendency to induce the orientation of molecular chains. However, under the restriction of the surrounding molecular chain segments, the molecular chains are unable to complete large-scale migration movements, and the conformation adjustment of the molecular chains is limited. Thus, the orientation and radius of gyration of the molecular chains change little.

(2) In the sheared pure PE system after the cessation of shear, the Brownian motion of the oriented molecular chains is active. The molecular chains overcome the viscous flow activation energy and relax dramatically. A significant conformation change rapidly occurs, from an oriented to a winded state, and so the conformation size of the molecular chains is markedly reduced.

(3) Under the adsorption and inhibition of the CNT in the sheared composite system, the conformation of the oriented chains stabilizes. Moreover, under the induction of the CNT, the molecular chains are further extended, orientation degree is further improved, and the arrangement is more regular and orderly. Compared with the isolated effects of shear flow and the CNT, the combination of the two has a more obvious effect on the conformation size and orientation degree of the molecular chains. Furthermore, the orderly arrangement of molecular chains is closer to a lattice structure, 
and the kinetic barrier to be crossed is relatively small, promoting the interface crystallization of the composite system.

(4) The RDF peak values of $\mathrm{C}$ and $\mathrm{H}$ atoms in the sheared composite system are higher than those in the static composite system, the van der Waals force increases, and the interface binding energy increases correspondingly, which indicates that the interface interaction between PE molecular chains and CNTs can be enhanced to some extent through interface crystallization. Moreover, by changing the process conditions of injection molding, the molecular chains can have different orientation degrees, so as to regulate the degree and morphology of crystallization at the interface.

Author Contributions: Conceptualization, data curation, and formal analysis, M.Z.; funding acquisition, Y.X.; investigation and methodology, M.Z.; project administration and supervision, Y.X.; visualization, M.Z.; writing-original draft, and review and editing, M.Z. and Y.X. All authors have read and agreed to the published version of the manuscript.

Funding: This study was supported by the National Natural Science Funds of China (No. 51365038) and College Science and Technology Project of the Government of Jiangxi Province (No. KJLD12058).

Acknowledgments: The financial support from the National Natural Science Funds of China (No. 51365038) and College Science and Technology Project of the Government of Jiangxi Province (No. KJLD12058) is gratefully acknowledged. We express our gratitude to the Shenzhen Cloud computing center for computing support and help. We also appreciate the efforts of the journal editor for editing this paper.

Conflicts of Interest: The authors declare no conflict of interest.

\section{References}

1. Iijima, S. Helical microtubules of graphitic carbon. Nature 1991, 354, 56-58. [CrossRef]

2. Salvetat, J.-P.; Briggs, G.A.D.; Bonard, J.-M.; Bacsa, R.R.; Kulik, A.J.; Stöckli, T.; Burnham, N.A.; Forró, L. Elastic and shear moduli of single-walled carbon nanotube ropes. Phys. Rev. Lett. 1999, 82, 944-947. [CrossRef]

3. Wong, E.W.; Sheehan, P.E.; Lieber, C.M. Nanobeam mechanics: Elasticity, strength, and toughness of nanorods and nanotubes. Science 1997, 277, 1971-1975. [CrossRef]

4. Berber, S.; Kwon, Y.-K.; Tomanek, D. Unusually high thermal conductivity of carbon nanotubes. Phys. Rev. Lett. 2000, 84, 4613-4616. [CrossRef]

5. Kim, P.; Shi, L.; Majumdar, A.; McEuen, P.L. Thermal transport measurements of individual multiwalled nanotubes. Phys. Rev. Lett. 2001, 87, 215502. [CrossRef]

6. Kim, B.; Lee, J.; Yu, I. Electrical properties of single-wall carbon nanotube and epoxy composites. J. Appl. Phys. 2003, 94, 6724-6728. [CrossRef]

7. Spitalsky, Z.; Tasis, D.; Papagelis, K.; Galiotis, C. Carbon nanotube-polymer composites: Chemistry, processing, mechanical and electrical properties. Prog. Polym. Sci. 2010, 35, 357-401. [CrossRef]

8. Ajayan, P.M.; Stephan, O.; Colliex, C.; Trauth, D. Aligned Carbon Nanotube Arrays Formed by Cutting a Polymer Resin-Nanotube Composite. Science 1994, 265, 1212-1214. [CrossRef]

9. Li, L.-L.; Zhang, X.-H.; Wang, Y.-L.; Guo, J.-H.; Zhang, S. Simulation of mechanical properties based on microstructure in polyethylene/montmorillonite nanocomposites. Acta Phys. Sin. 2016, 65, 196202.

10. Yu, B.; Fu, S.; Wu, Z.; Bai, H.; Ning, N.; Fu, Q. Molecular dynamics studies of interfacial crystallization behaviors in polyethylene/carbon nanotube composites. RSC Adv. 2015, 5, 102219-102227. [CrossRef]

11. Wei, C.; Srivastava, D.; Cho, K. Structural Ordering in Nanotube Polymer Composites. Nano Lett. 2004, 4, 1949-1952. [CrossRef]

12. Zhang, D.; Yang, H.; Liu, Z.; Liu, A.; Li, Y. Interfacial interaction between polypropylene and nanotube: A molecular dynamics simulation. J. Mol. Struct. 2017, 1144, 260-264. [CrossRef]

13. Choi, H.K.; Jung, H.; Oh, Y.; Hong, H.; Yu, J.; Shin, E.S. Interfacial effects of nitrogen-doped carbon nanotubes on mechanical and thermal properties of nanocomposites: A molecular dynamics study. Compos. Part B Eng. 2019, 167, 615-620. [CrossRef]

14. Hu, Y.; Ding, J.L. Effects of morphologies of carbon nanofillers on the interfacial and deformation behavior of polymer nanocomposites-A molecular dynamics study. Carbon 2016, 107, 510-524. [CrossRef] 
15. Sun, S.; Chen, S.; Weng, X.; Shan, F.; Hu, S. Effect of carbon nanotube addition on the interfacial adhesion between graphene and epoxy: A molecular dynamics simulation. Polymers 2019, 11, 121. [CrossRef]

16. Syed, F.; Zainuddin, S.; Willis, A.; Hosur, M.; Jeelani, S. Crosslinking and interfacial behavior of carboxylic functionalized carbon nanotube Epon nanocomposites: A molecular dynamic simulation approach. SN Appl. Sci. 2019, 1, 1423. [CrossRef]

17. Yang, J.-S.; Yang, C.-L.; Wang, M.-S.; Chen, B.-D.; Ma, X.-G. Crystallization of alkane melts induced by carbon nanotubes and graphene nanosheets: A molecular dynamics simulation study. Phys. Chem. Chem. Phys. 2011, 13, 15476-15482. [CrossRef]

18. Yang, H.; Chen, Y.; Liu, Y.; Cai, W.S.; Li, Z.S. Molecular dynamics simulation of polyethylene on single wall carbon nanotube. J. Chem. Phys. 2007, 127, 094902. [CrossRef]

19. Liu, J.; Zhao, L.; Lu, Z.-Y.; Li, Z.-S. Molecular dynamics simulation of adsorption of a polyethylene chain on carbon nanotube. Chem. J. Chin. Univ. 2008, 29, 2389-2392.

20. Karatrantos, A.; Composto, R.J.; Winey, K.I.; Clarke, N. Structure and Conformations of Polymer/SWCNT Nanocomposites. Macromolecules 2011, 44, 9830-9838. [CrossRef]

21. Solomon, N.; Solomon, I.; Sanduleac, E. Material flow influence on the quality of molded parts. Polym. Bull. 2019, 76, 5981-6000. [CrossRef]

22. Parodi, E.; Peters, G.W.M.; Govaert, L.E. Structure-properties relations for polyamide 6, Part 2: Influence of processing conditions during injection moulding on deformation and failure kinetics. Polymers 2018, 10, 779. [CrossRef]

23. Minoia, A.; Chen, L.; Beljonne, D.; Lazzaroni, R. Molecular modeling study of the structure and stability of polymer/carbon nanotube interfaces. Polymer 2012, 53, 5480-5490. [CrossRef]

24. Wang, J.; Cao, C.; Chen, X.; Ren, S.; Chen, Y.; Yu, D.; Chen, X. Orientation and dispersion evolution of carbon nanotubes in ultra high molecular weight polyethylene composites under extensional-shear coupled flow: A dissipative particle dynamics study. Polymers 2019, 11, 154. [CrossRef]

25. Zhou, B.; Luo, W.; Yang, J.; Duan, X.; Wen, Y.; Zhou, H.; Chen, R.; Shan, B. Simulation of dispersion and alignment of carbon nanotubes in polymer flow using dissipative particle dynamics. Comput. Mater. Sci. 2017, 126, 35-42. [CrossRef]

26. Yang, H.; Liu, Y.; Zhang, H.; Li, Z.-S. Diffusion of single alkane molecule in carbon nanotube studied by molecular dynamics simulation. Polymer 2006, 47, 7607-7610. [CrossRef]

27. Sun, H. COMPASS: An ab Initio Force-Field Optimized for Condensed-Phase Applications Overview with Details on Alkane and Benzene Compounds. J. Phys. Chem. B 1998, 102, 7338-7364. [CrossRef]

28. Xu, J.-Z.; Zhong, G.-J.; Hsiao, B.S.; Fu, Q.; Li, Z.-M. Low-dimensional carbonaceous nanofiller induced polymer crystallization. Prog. Polym. Sci. 2014, 39, 555-593. [CrossRef]

29. Durand, M.; Meyer, H.; Benzerara, O.; Baschnagel, J.; Vitrac, O. Molecular dynamics simulations of the chain dynamics in monodisperse oligomer melts and of the oligomer tracer diffusion in an entangled polymer matrix. J. Chem. Phys. 2010, 132, 194902. [CrossRef]

30. Fujiwara, S.; Sato, T. Molecular dynamics simulations of structural formation of a single polymer chain: Bond-orientational order and conformational defects. J. Chem. Phys. 1997, 107, 613-622. [CrossRef]

(C) 2020 by the authors. Licensee MDPI, Basel, Switzerland. This article is an open access article distributed under the terms and conditions of the Creative Commons Attribution (CC BY) license (http://creativecommons.org/licenses/by/4.0/). 